\title{
SCHUR CONVEXITY AND SCHUR-GEOMETRICALLY CONCAVITY OF GENERALIZED EXPONENT MEAN
}

\author{
DA-MAO LI AND HUAN-NAN SHI
}

Abstract. The monotonicity, the Schur-convexity and the Schur-geometrically convexity with variables $(x, y)$ in $\mathbb{R}_{++}^{2}$ for fixed $a$ of the generalized exponent mean $I_{a}(x, y)$ is proved. Besides, the monotonicity with parameters $a$ in $\mathbb{R}$ for fixed $(x, y)$ of $I_{a}(x, y)$ is discussed by using the hyperbolic composite function. Furthermore, some new inequalities are obtained.

Mathematics subject classification (2000): 26D15, 26A51.

Keywords and phrases: Generalized exponent mean, monotonicity, Schur-convexity, Schur-geometrically concavity inequality, hyperbolic function.

\section{REFERENCES}

[1] K. B. Stolars Ky, Generalizations of the logarithmic mean, Math. Mag., 48, 2 (1975), 87-92.

[2] Huan-NAn ShI, Shan-He Wu AND Feng QI, An alternative note on the Schur-convexity of the extended mean values, Mathematical Inequalities and Applications, 9, 2 (2006), 219-224.

[3] Bo-YInG WANG, Foundations of Majorization Inequalities, Beijing Normal Univ. Press, Beijing, China, 1990. (Chinese)

[4] A. M. MaRShall AND I. OlKIn, Inequalities:theory of majorization and its application, New York: Academies Press, 1979.

[5] Xiao-Ming Zhang, Geometrically Convex Functions, Hefei: An'hui University Press, 2004.(Chinese)

[6] Constantin P. Niculescu, Convexity According to the Geometric Mean, Mathematical Inequalities \& Applications, 3, 2 (2000), 155-167.

[7] Da-Mao Li, Chun Gu And Huan-Nan Shi, Schur Convexity of the Power-Type Generalization of Heronian Mean, Mathematics in practice and theory, 36, 9 (2006), 387-390. (Chinese) 